\section{Ectopic ATP synthase $\beta$ subunit proteins on human leukemia cell surface interact with platelets by binding glycoprotein IIb}

The role of platelets in metastasis of solid tumors has been extensively studied, and proposed mechanisms include both platelet-cancer aggregation (PCA) ${ }^{1}$ and production of soluble factors/microvesicles/exosomes targeting either cancer cells or their microenvironment. Reported molecular interactions during PCA include direct ligand-receptor pairs, like podoplanin-CLEC2 and P-selectin-PSGL-1, or indirect ones like those mediated by crosslinking of glycoproteins (GPs) on both platelets and cancer cells to the same polymerized molecules such as collagens, von Willebrand factor, fibrinogens, etc. ${ }^{2}$ However, the interactions between platelets and another class of malignancies that more likely encounter platelets, namely leukemia, have been less well-defined. Recent encouraging reports have demonstrated that platelets might also play a role in the pathogenesis and prognosis of leukemia. ${ }^{3-5}$ Using murine leukemia cell lines, we showed that murine platelets regulated leukemia cell survival $^{6}$ or response to therapeutical chemical, and that this was potentially mediated via platelet-leukemia aggregation (PLA) (Online Supplementary Figure S1). ${ }^{7}$ We later observed that some human leukemia cell lines also bound platelets. At the same time, in an effort to define the epitope recognized by a widely-used monoclonal anti-human GPIIb antibody (e.g. SZ22), ${ }^{8}$ we found that

A

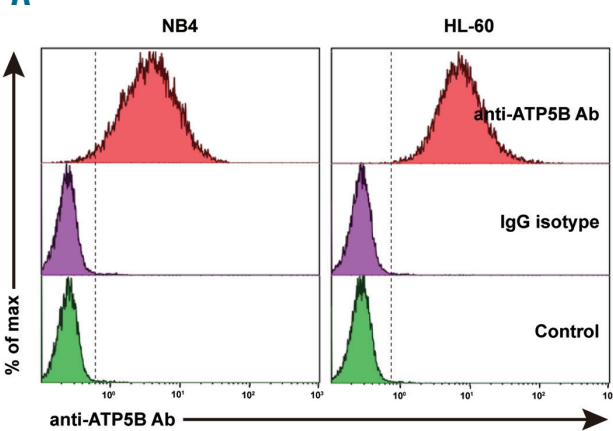

B

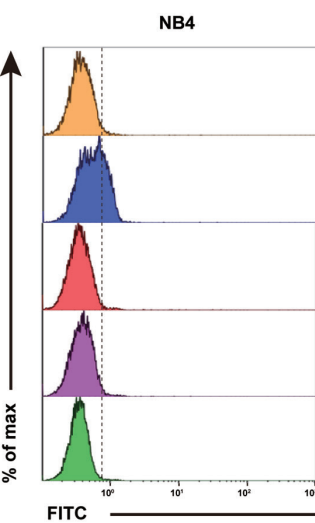

D
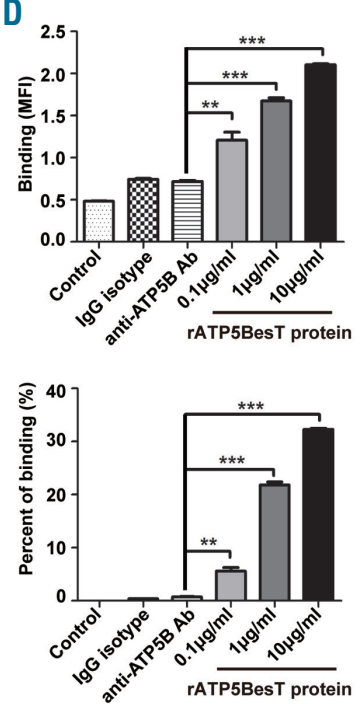

E
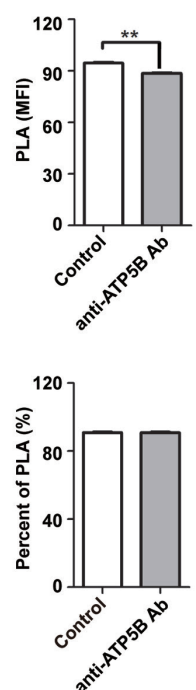

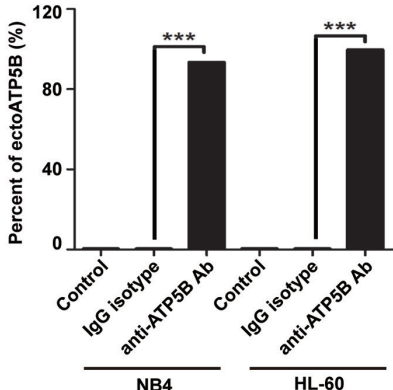

C
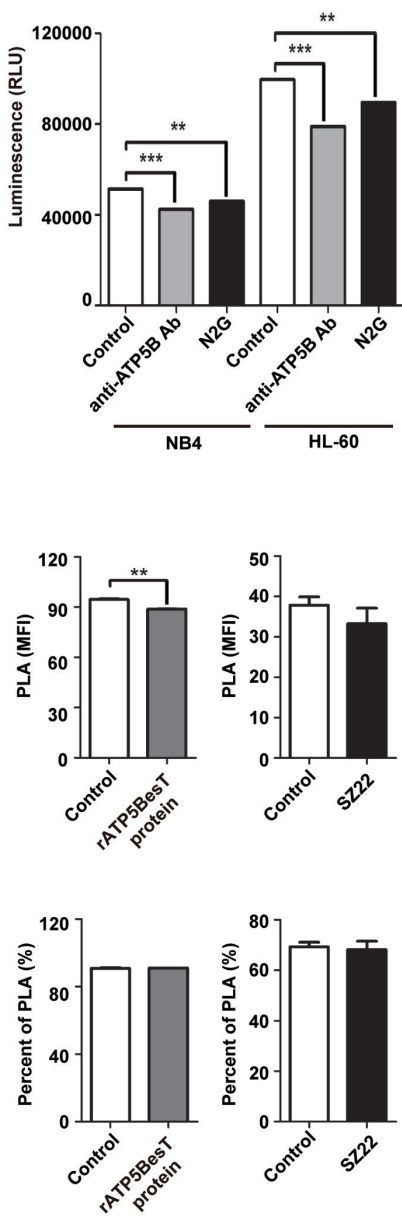

Figure 1. Identification of ectopic ATP5B as N2G-epitope counterpart on NB4 cells. (A) Flow cytometry demonstrated expression of ectopic ATP5B on the outer surface of NB4 and $\mathrm{HL}-60$ cells. NB4 or $\mathrm{HL}-60$ cells were incubated with anti-ATP5B antibodies $(5 \mu \mathrm{g} / \mathrm{mL})$ at $4^{\circ} \mathrm{C}$ for 30 minutes $(\mathrm{min})$, followed by staining with PE-conjugated donkey-anti-rabbit antibodies. (B) Pre-treatment with antiATP5B antibodies $(5 \mu \mathrm{g} / \mathrm{mL})$ at $4^{\circ} \mathrm{C}$ for 30 min inhibited N2G-peptides (10 uM) binding onto NB4 or HL-60 cells as measured by flow cytometry. (C) N2G-peptides or anti-ATP5B antibodies decreased ATP synthesis on NB4 or HL-60 cell surface. Cells were incubated with N2G-peptides $(10 \mu \mathrm{M})$ or anti-ATP5B antibodies $(5 \mu \mathrm{g} / \mathrm{mL})$ at $37^{\circ} \mathrm{C}$ for 1 hour in HEPES-MgCl $l_{2}$ buffer, and then switched to fresh $\mathrm{MgCl}_{2}$ buffer containing 100 uM ADP for 15 seconds (sec). Supernatant was collected by $1200 \mathrm{rpm}$ centrifugation. ATP contents were measured with an Enhanced ATP Assay Kit and final readouts were obtained on a plate reader. (D) ATP5B analog rATP5BesT protein-bound human platelets. Washed and resting human platelets were incubated with rATP5BesT proteins $(0.1,1,10 \mu \mathrm{g} / \mathrm{mL})$ at room temperature for $30 \mathrm{~min}$, and anti-ATP5B antibodies were added to $5 \mathrm{\mu g} / \mathrm{mL}$ for another $30 \mathrm{~min}$. Donkey-anti-rabbit IgG/PE were used as secondary antibody for flow cytometry. Mean fluorescence intensity (MFI) of all platelets (top panel) or percentages of platelets judged as positive staining (bottom panel) were calculated. For controls, rATP5BesT was omitted, or anti-ATP5B antibodies were substituted by isotype control. (E) Anti-ATP5B antibodies and rATP5BesT slightly but statistically significantly inhibited platelet aggregation onto NB4 cells. Washed and resting human platelets were labeled with Calcein $(5 \mathrm{\mu g} / \mathrm{mL}$ ) and incubated with rATP5BesT protein $(10 \mu \mathrm{g} / \mathrm{mL})$ or SZ22 antibodies (1 $\mu \mathrm{g} / \mathrm{mL}$ ) for $30 \mathrm{~min}$, and then added to NB4 cells for $30 \mathrm{~min}$. Or the cells were pre-treated with anti-ATP5B antibodies $(5 \mathrm{ug} / \mathrm{mL})$ at $4{ }^{\circ} \mathrm{C}$ for $30 \mathrm{~min}$ before co-culture with Calceinlabeled platelets. Flow cytometry was used to compare the intensity of cells (with bound platelets) between treatments and controls. All experiments were performed three times with similar results, and representative experiments of these are shown. For numeric data, mean \pm Standard Deviation (SD) was obtained from two (A-D) or four (E) duplicates in each treatment. Student $t$-test, $* * P<0.01$; $* * * P<0.001$. PLA: platelet-leukemia. 
the epitope recognized by SZ22 was likely a surface dock for certain partners. Given this, and continuing our research, we discovered that this platelet GPIIb epitope interacted with ectopic ATP synthase subunit $\beta$ (ectoATP5B) on the leukemia cell surface. Furthermore, utilizing a mimetic peptide corresponding to this epitope, cytotoxic peptides could be delivered specifically to ectoATP5B-positive cells, hence achieving targeted cell killing.

First, the epitope recognized by SZ22 was identified by using a phage display methodology. After three rounds of panning the Ph.D-12 phage library against immobilized SZ22 antibodies, 12 phage clones were sequenced. Eleven of them identified a conserved 8-amino acid motif that was homologous with mature human GPIIb at 833-
840, namely NPLKVDWG, herein referred to as N2G (Online Supplementary Figure S2A). To test actual binding of SZ22 antibodies with sequences containing N2G epitope, mimetic N2G-peptides and L835-W839 switched controls (i.e. NPWKVDLG, NsG-peptides) were chemically synthesized, with or without FITC or biotin modification at the N-terminals. Binding of biotin-N2G-peptides with immobilized SZ22 antibodies in ELISA or SZ22 binding onto denatured platelet proteins in blot membrane was dose-dependently blocked by N2G-peptides, but not by NsG-peptides in either situation (Online Supplementary Figure S2B and C). SZ22 also bound a recombinant GPIIb fragment corresponding to the Calf2 domain of GPIIb, but not its mutation form in which L835-W839 was switched like in NsG-peptides (Online
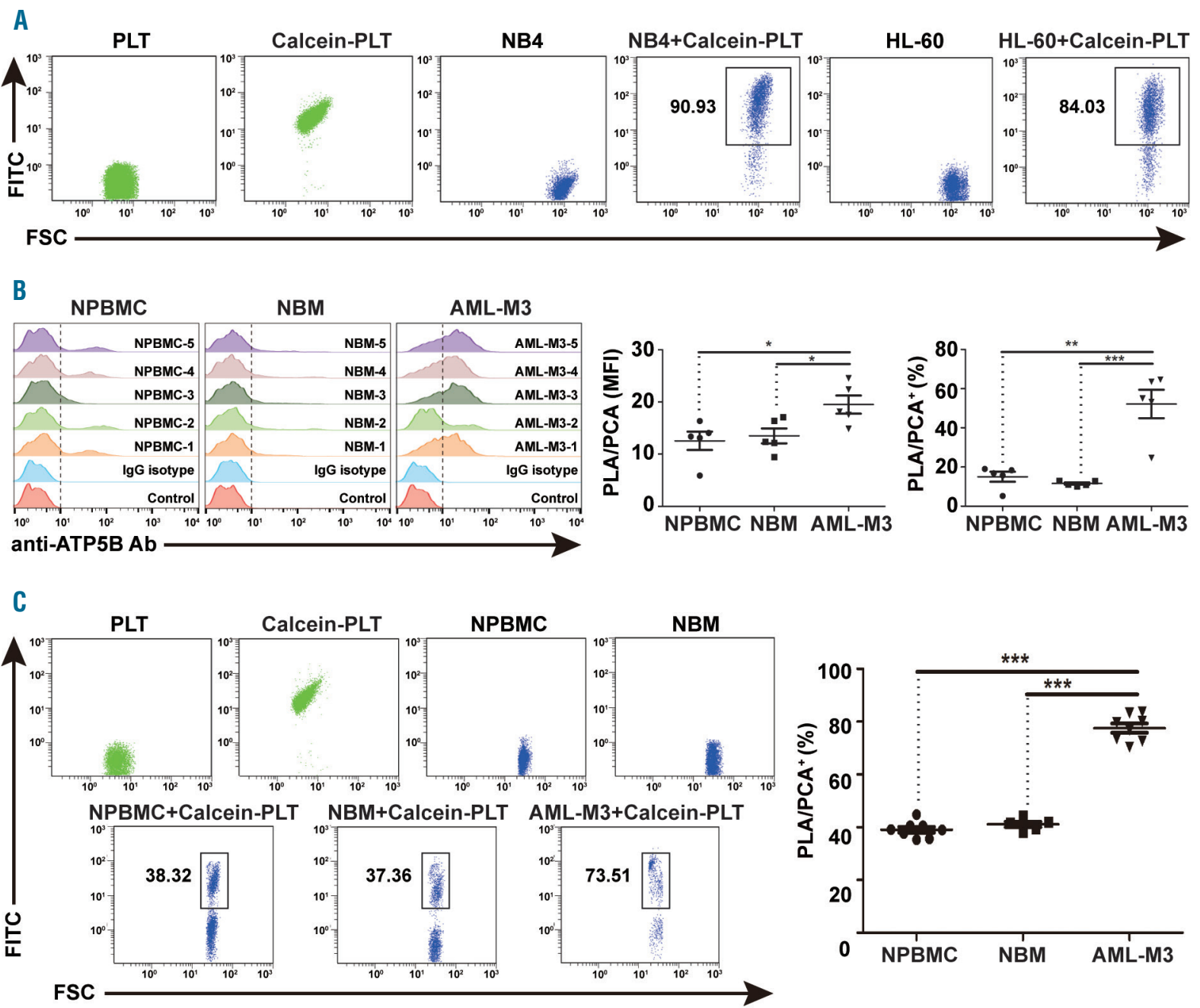

Figure 2. Binding of human platelets (PLT) onto leukemic cells expressing ectoATP5B and bone marrow cells of acute myeloid leukemia (AML)-M3 patients. (A) Purified normal human platelets were labeled with Calcein and cultured with NB4 or $\mathrm{HL}-60$ cells at $37^{\circ} \mathrm{C}$ for 30 minutes (min) before running on a flow cytometer. Shows one of the three repeats that gave similar results. (B) Peripheral blood mononuclear cells (PBMC) from healthy donors (NPBMC, $n=5$ ), or bone marrow cells from donors without any hematogenous disorders (NBM, $n=5$ ) or from patients with AML-M3 ( $=5$ ) were incubated with anti-ATP5B ( 5 g/ $m L$ ) at $4{ }^{\circ} \mathrm{C}$ for $30 \mathrm{~min}$ followed by incubation in PE donkey anti-rabbit IgG. Cells from one donor in each group were left unstained or stained with IgG isotype control antibody for use as controls. The mean fluorescence intensity of all cells and percentage of ectoATP5B-positive cells were calculated. Mean \pm Standard Deviation (SD) was obtained from five samples. (C) Binding of Calcein-labeled resting platelets with healthy PBMC ( $n=8$ ) or bone marrow cells from normal $(n=5)$ or $A M L-$ M3 $(n=8)$ donors was measured as (A). Cells from one NPMBC donor and one NBM donor were left unstained for use as controls. (Left) Shows one sample in each group. (Right) Summary of all samples in three groups. Note: samples of five NPBMC donors and five AML-M3 patients of (B) were also used for (C). 
A

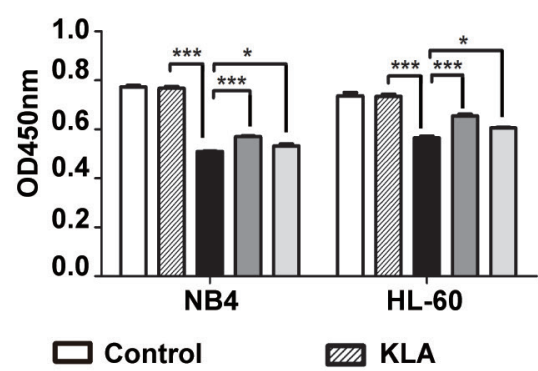

B

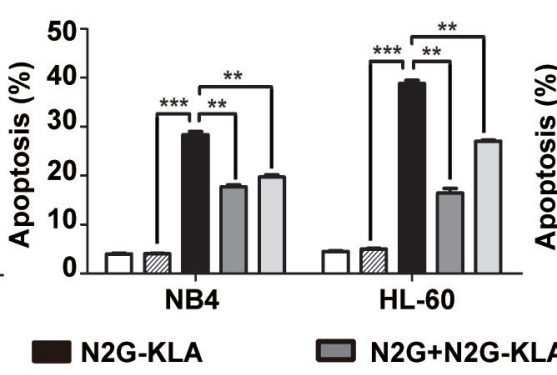

$c$

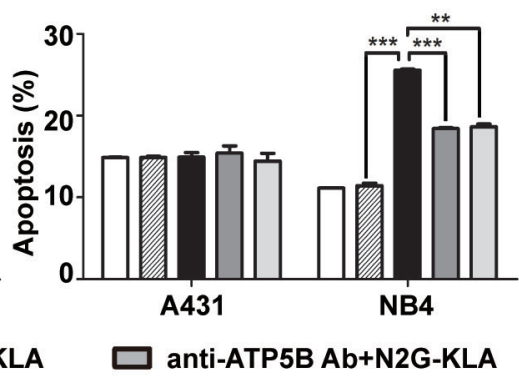

D

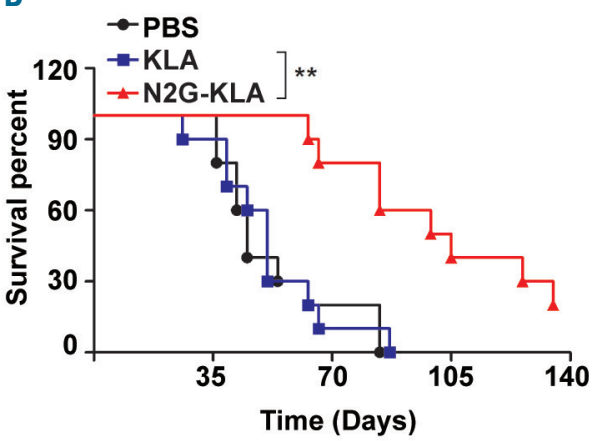

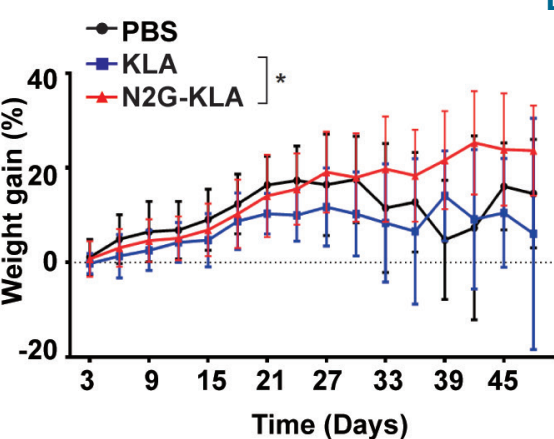

E

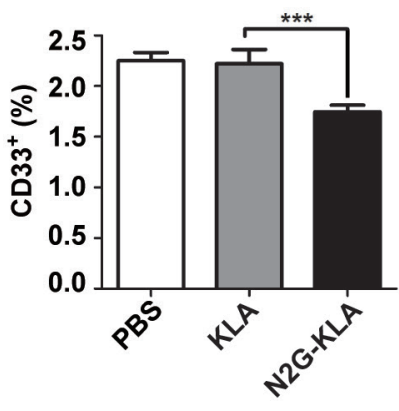

Figure 3. N2G-guided targeting cytotoxicity of ectoATP5B-positive cells in vitro and in vivo. (A and B) NB4 cells and HL-60 cells, pre-treated or not with $60 \mu \mathrm{M}$ N2G-peptides or $50 \mu \mathrm{g} / \mathrm{mL}$ anti-ATP5B antibodies at $37^{\circ} \mathrm{C}$ for 1 hour $(\mathrm{h})$, were cultured with $30 \mu \mathrm{M}$ N2G-KLA or control KLA peptides for $24 \mathrm{~h}$ before being measured for cell growth with CCK8 colorigenic methods (A) or for apoptosis with Annexin-V/7AAD staining and FACS (B). (C) NB4 cells were labeled with 20 nM CFSE and mixed with an equal number of A431 cells, then subjected to pre-treatment with N2G-peptides or anti-ATP5B antibodies followed by treatment with N2G-KLA or KLA peptides and apoptosis measurement as in (B). ( $D$ and E) NB4 cells $\left(4 \times 10^{6}\right)$ were injected into NOD-SCID mice ( $n=10$ each group) via tail vein on day 1 to set up a xenograft leukemia model. Then on each third day through the first month, the mice were given $200 \mu \mathrm{g}$ N2G-KLA or KLA peptides or same volume of PBS buffer via tail vein. Body weight and survival were monitored till the end of experiment. Survival data are expressed as the Kaplan-Meier survival curve, and body weight changes (as the ratio of gained weight to original weight of each mouse on the day of NB4 inoculation) was analyzed using Least-Significant Difference analysis (D). In another experimental setting, mice were sacrificed three days after the last peptide dosing, and total bone marrow cells were harvested from femurs and stained with anti-human CD33 antibody to identify NB4 cells. After flow cytometry, percentage of NB4 cells in total nuclear cells of bone marrow were obtained $(\mathrm{E})$. One representative of three $(\mathrm{A}-\mathrm{C})$ or two $(\mathrm{D}$ and $\mathrm{E})$ independent experiments are shown. $* P<0.05$; $\star \star P<0.01$; $\star * * P<0.001$.

Supplementary Figures S2D and S3). Binding of intact platelets by SZ22, as measured using FACS, was blocked by N2G-peptides (Online Supplementary Figures S2E), confirming that the N2G epitope on GPIIb was the physiological site recognized by SZ22 antibodies. In the crystal 3D structure of GPIIbIIIa (PDB 3FCS ${ }^{9}$ ), N2G epitope was a rigid segment followed by a non-crystallized loop, both stretching on the surface of the Calf2 domain (Online Supplementary Figure S4), thus allowing access by large molecules like antibody or physiological partners. This area hosts several aggregation-related platelet antigenic haplotypes, including Max-aa837, ${ }^{10} \mathrm{Cab3}-\mathrm{aa} 841,{ }^{11}$ and Bak-aa843, ${ }^{12}$ suggesting that the N2G epitope might be involved in GPIIbIIIa transfiguration or platelet aggregation. Indeed, when added to platelet preparations in the presence of $10 \mu \mathrm{M} \mathrm{ADP}$ or $0.05 \mathrm{U} / \mathrm{mL}$ thrombin, N2Gpeptides slightly inhibited GPIIbIIIa complex formation on the platelet surface (Online Supplementary Figure S2F), and significantly reduced platelet aggregation (Online Supplementary Figure $S 2 G$ ) and clot retraction (Online Supplementary Figure S2H). NsG-peptides did not show a significant effect in any of these functional assays (Online Supplementary Figure S2E-H). These data suggested that the GPIIb 833-840 epitope was involved in the activation and aggregation of platelets induced by agonists, and that mimetic N2G-peptides can be utilized to manipulate platelet-platelet aggregation.

To check if the N2G epitope was also involved in PLA or PCA, we measured the hypothetical binding of FITC$\mathrm{N} 2 \mathrm{G}$-peptides onto cancerous or leukemic cell lines. In general, leukemic cells bound more FITC-N2G-peptides than non-leukemic ones (Online Supplementary Figure S5A and data not shown), and human acute promyelocytic leukemia NB4 was selected as a representative line for most subsequent studies. To identify receptor(s) on NB4 cells for N2G-peptides, membrane proteins prepared from NB4 cells were subjected to pull-down procedure against N2G-peptides-conjugated matrix beads. Mass spectrum assay of the proteins recovered from the beads demonstrated that ATP5B gave the highest matching scores (Online Supplementary Table S1), and that this was, therefore, the most probable counterpart on NB4 cells that interacts with N2G epitope on GPIIb molecules. Western blotting of the pulled-down fractions confirmed the existence of ATP5B in them (Online Supplementary Figure $S 5 B$ ). Since some cancer cells or normal cells (e.g. hepatocytes, vascular endothelial cells) express ATP synthase or ATP5B on their surface, ${ }^{13}$ we then verified this with FACS, and confirmed the presence of ectoATP5B on NB4 and HL-60 (Figure 1A), but it was essentially absent on other cancer cells (Online Supplementary Figure S5D). To confirm the specificity of ectoATP5B proteins in these 
studies, a recombinant ATP5B analog (rATP5BesT) (Online Supplementary Figure S6) containing a tandem of main predicted B epitopes on ATP5B was obtained via engineered expression in $E$. coli, and polyclonal antirATP5BesT antibodies were produced in rabbits, both of which were used for blocking N2G-peptides binding. As expected, the binding of FITC-N2G-peptides with NB4 cells was blocked by anti-ATP5B antibodies (Figure 1B), and such binding decreased ATP production on the NB4 cell surface (Figure $1 \mathrm{C}$ ), as seen with solid tumor cells. ${ }^{14}$ These data suggested that ectoATP5B on NB4 cell surface could function as a direct 'receptor' of N2G-peptides. We next investigated whether this finding had any physiological significance, namely if platelet GPIIb posed a physiological partner of ectoATP5B on leukemia cells. The results that $\mathrm{AATP} 5 \mathrm{Bes} \mathrm{T}$ proteins bound onto platelets in a dose-dependent manner (Figure 1D) strongly suggested that if a leukemia or cancer cell does express ectoATP5B, it would aggregate with platelets via the ectoATP5B-GPIIb bridge. This was confirmed not only with leukemic cells lines, but also with cells from bone marrow cells from human leukemia patients (Figure 2). Though neither N2G-peptides nor anti-ATP5B antibodies had very much effect on NB4 cell behavior (Online Supplementary Figure S7), when rATP5BesT proteins or anti-ATP5B antibodies were added to the co-culture system of platelets and leukemia cells, a statistically significant, though slight, decrease of PLA was observed for NB4 cells (Figure 1E), indicating that GPIIb-ectoATP5B interaction contributed to the affinity or physical forces causing PLA. In support of such hypothetical physical attractions between GPIIb and ectoATP5B, structural modeling showed that, when the surface of whole ATP synthase was screened blindly for potential binding sites of N2G-peptides, 44 of the top 50 best poses were located onto a grove on the surface of one of the ATP5B chain (chain_E) (Online Supplementary Figure S8A). Considering that there were three ATP5B subunits in all (1E79_D, $1 E 79\left[E, 1 E 79 \_F\right)$ in the intact ATP synthase complex, the preferred binding of N2G-peptides onto chain E only implied a strict requirement for the proposed GPIIbATP5B interactions. Using that site as a hinge for docking, a reliable GPIIb-ATP synthase complex model was obtained (Online Supplementary Figure S8B), thus providing support for an actual GPIIb-ectoATP5B conjugation from a point of structural biology.

Finally, we checked if the N2G-ectoATP5B pathway could be exploited to develop a targeting therapeutic protocol. Using various linkers (data not shown), a complex peptide NPLKVDWG-LP-KLAKLAKKLAKLAK (N2GKLA) was tried out, in which the mitochondria-targeting proapoptotic KLAKLAKKLAKLAK ${ }^{15}$ domain should function as cytotoxin. When added to the culture of NB4 or HL-60 cells, N2G-KLA peptides decreased overall cell expansion and induced cell apoptosis, both effects being hampered by the previous addition of N2G-pepides or anti-ATP5B antibodies (Figure 3A and B). Interestingly, when NB4 cells were mixed with ectoATP5B-negative A431 cells in culture, N2G-KLA induced apoptosis in NB4 cells only (Figure 3C). In all these studies, control peptides NsG-KLA did not manifest a significant effect (data not shown). Then a leukemia-like model was established in NOD-SCID mice with NB4 cells and were treated with N2G-KLA or KLA peptides every third day from day 4. N2G-KLA peptides significantly alleviated weight loss and prolonged survival of model mice (Figure 3D). At the end of the experiments, the percentage of leukemic cells in the bone marrow of N2G-KLA-treated mice was less than that in control mice (Figure $3 \mathrm{E}$ ), demonstrating a protective effect of N2G-KLA peptides in those leukemia models.

In summary, current data revealed GPIIb-ectoATP5B interaction as a mechanism of PLA. Based on current knowledge of ectoATP5B in solid cancers, we hypothesized that the GPIIb-ectoATP5B duo should also function in those ectoATP5B-positive cancers, and investigated the significance of current findings in PLA/PCA as a whole. Compared with known interacting pairs contributing to PLA/PCA, GPIIb-ectoATP5B interaction is unique in that it occurs when platelets are at rest and GPIIb is in 'close' configuration. This feature allows circulating platelets to recognize ectoATP5B-bearing leukemia/cancer cells without previous activation. Although N2G-peptides binding onto ectoATP5B alone has little effect on leukemia cells (Online Supplementary Figure S7), and this interaction only contributes minor mechanical forces to PLA/PCA (Figure 1E), there is a possibility that GPIIb-ectoATP5B interaction could initiate a cascade that has an extremely strong effect on PLA/PCA in in vivo conditions. Also, as seen using N2G-KLA peptides, a N2G-sequence might be utilized to deliver cytotoxins to ectoATP5B-positive leukemia/cancer cells, thus providing targeted therapy. Future studies should confirm ectoATP5B expression in larger patient samples, and check the potential side-effects of proposed N2G-guided toxins in normal tissues expressing ectoATP5B.

Ting Wang, ${ }^{1}$ Ying Shen, ${ }^{1}$ Yuanyuan Li, Beibei Wang, ${ }^{2}$ Benfang Wang, ${ }^{1}$ Depei Wu, ${ }^{3}$ Changgeng Ruan ${ }^{1}$ and Yiqiang Wang'

'MOH Key Lab of Thrombosis and Hemostasis, Collaborative Innovation Center of Hematology, Jiangsu Institute of Hematology, The First Affiliated Hospital of Soochow University, Medical College, Soochow University, Suzhou; ${ }^{2}$ Center for Informational Biology, School of Life Science and Technology, University of Electronic Science and Technology of China, Chengdu and Institute of Hematopoietic Stem Cell Transplantation, Department of Hematology, The First Affiliated Hospital of Soochow University, Medical College, Soochow University, Suzhou, P. R. China

Acknowledgments: the authors would like to thank Drs. Lijun Xia and Heyu Ni for helpful discussions, Jinling Ning and Zhenjiang Sun for technical assistance.

Funding: this work was supported by a grant from the National Natural Science Foundation of China (81571544), a starting package from The First Affiliated Hospital of Soochow University, the Jiangsu Provincial Special Program of Medical Science (BL2012005), and the Jiangsu Province's Key Medical Center Program.

Correspondence: YIQIANG WANG.yiqiangwang@suda.edu.cn doi:10.3324/haematol.2019.216390

Information on authorship, contributions, and financial \& other disclosures was provided by the authors and is available with the online version of this article at www. haematologica.org.

\section{References}

1. Stegner D, Dutting S, Nieswandt B. Mechanistic explanation for platelet contribution to cancer metastasis. Thromb Res. 2014;133 Suppl 2:S149-157

2. Estevez B, Du X. New Concepts and Mechanisms of Platelet Activation Signaling. Physiology (Bethesda). 2017;32(2):162-177.

3. Bruserud $O$, Foss B, Hervig T. Effects of normal platelets on proliferation and constitutive cytokine secretion by human acute myelogenous leukaemia blasts. Platelets. 1997;8(6):397-404.

4. Yamazaki E, Kanamori $\mathrm{H}$, Itabashi $\mathrm{M}$, et al. Hyper-recovery of platelets after induction therapy is a predictor of relapse-free survival in acute myeloid leukemia. Leuk Lymphoma. 2017;58(1):104-109.

5. Mangaonkar A, Xu H, Mohsin J, et al. Prognostic value of complete remission with superior platelet counts in acute myeloid leukemia. J Community Support Oncol. 2016;14(2):66-71. 
6. Wang Y, Zhang H. Platelet-induced inhibition of tumor cell growth. Thromb Res. 2008;123(2):324-330.

7. Hu Y, Shao L, Zhao L, Shen Y, Wu K, Wang Y. [Platelets decreas the sensititity of leukemia cell L1210 to multiple druges via actibiting the AKT and ERK signaling pathway]. Zhongguo Shi Yan Xue Ye Xue Za Zhi. 2016;24(5):1489-1494.

8. Jallu V, Diaz-Ricart M, Ordinas A, Pico M, Vezon G, Nurden AT. Two human antibodies reacting with different epitopes on integrin beta 3 of platelets and endothelial cells. Eur J Biochem. 1994;222(3):743-751.

9. Zhu J, Luo BH, Xiao T, Zhang C, Nishida N, Springer TA. Structure of a complete integrin ectodomain in a physiologic resting state and activation and deactivation by applied forces. Mol Cell. 2008; 32(6):849-861.

10. Noris P, Simsek S, de Bruijne-Admiraal LG, et al. Max(a), a new lowfrequency platelet-specific antigen localized on glycoprotein IIb, is associated with neonatal alloimmune thrombocytopenia. Blood. 1995;86(3):1019-1026.
11. Jallu V, Bertrand G, Bianchi F, Chenet C, Poulain P, Kaplan C. The alphaIIb p.Leu841Met (Cab3(a+) ) polymorphism results in a new human platelet alloantigen involved in neonatal alloimmune thrombocytopenia. Transfusion. 2013;53(3):554-563.

12. Lyman S, Aster RH, Visentin GP, Newman PJ. Polymorphism of human platelet membrane glycoprotein IIb associated with the Baka/Bakb alloantigen system. Blood. 1990;75(12):2343-2348.

13. Taurino F, Gnoni A. Systematic review of plasma-membrane ectoATP synthase: A new player in health and disease. Exp Mol Pathol. 2018;104(1):59-70.

14. Arakaki N, Nagao T, Niki R, et al. Possible role of cell surface H+ ATP synthase in the extracellular ATP synthesis and proliferation of human umbilical vein endothelial cells. Mol Cancer Res. 2003; 1(13):931-939

15. Ellerby HM, Arap W, Ellerby LM, et al. Anti-cancer activity of targeted pro-apoptotic peptides. Nat Med. 1999;5(9):1032-1038. 